Revue

Revue de l'histoire des religions

de Ihistoire des religions

\title{
Universitas et natio. La fides des juifs dans les villes ibériques à la fin du Moyen Âge
}

Universitas and Natio: The fides of Jews in Iberian Cities in the Late Middle Ages

\section{Claude Denjean}

\section{(2) OpenEdition}

\section{Journals}

Édition électronique

URL : http://journals.openedition.org/rhr/8746

DOI : ERREUR PDO dans /localdata/www-bin/Core/Core/Db/Db.class.php L.34 : SQLSTATE[HYOOO]

[2006] MySQL server has gone away

ISSN : 2105-2573

\section{Éditeur}

Armand Colin

\section{Édition imprimée}

Date de publication : 1 juin 2017

Pagination : 337-358

ISBN : 978-2-200-93126-1

ISSN : 0035-1423

Référence électronique

Claude Denjean, « Universitas et natio. La fides des juifs dans les villes ibériques à la fin du Moyen Âge », Revue de l'histoire des religions [En ligne], 2 | 2017, mis en ligne le 01 juin 2019, consulté le 08 janvier 2021. URL : http://journals.openedition.org/rhr/8746 ; DOl : https://doi.org/10.4000/rhr.8746 


\section{Universitas et natio La fides des juifs dans les villes ibériques à la fin du Moyen Âge}

Si les travaux antérieurs ont établi qu'une nation juive n'était pas explicitement considérée, l'observation des hommes d'affaires démontre que les juifs ne sont pas une natio étrangère, tout en présentant des caractères comparables aux Italiens des «nations marchandes». Le jeu entre les stratégies marchandes et les représentations de l'usure déterminent les caractères économiques de l'appartenance nationale, déterminant des mécanismes à la fois intégrateurs et porteurs en germe d'exclusion, selon une évolution non linéaire et dialectique. Cette nation à l'ancienne sans natio, paradoxale mais déclinée au présent, se retrouve dans l'espace textuel de la nova d'Esther ou du récit de voyage, plus ressentie que territoriale, politique ou juridique et certainement pas univoque.

\section{Universitas and Natio:}

\section{The fides of Jews in Iberian Cities in the Late Middle Ages}

While previous works have established that the Jewish nation was not explicitly considered as such, examining the case of businessmen can reveal that the Jews were not a foreign natio and demonstrated a character comparable to that of the Italians of the "merchant nations". Interaction between business strategies and representations of usury determined the economic characteristics of national affiliation, thus determining mechanisms that could at the same time favor integration or lead to exclusion, following a non-linear, dialectic evolution. This oldfashioned nation without a natio, paradoxical but declined in the present, can be found in the textual space of the nova of Esther or of the travel narrative, was more affective than territorial, political or juridical, and was certainly not unequivocal. 
Si nous exprimons de manière lapidaire les conclusions de la réflexion conduite pour le colloque de la Société des Médiévistes de l'Enseignement Supérieur Public, il n'y a pas véritablement une nation juive au Moyen Âge ${ }^{1}$. Quant à découvrir des témoignages qui emploieraient le terme «natio » pour désigner les juifs méridionaux et ibériques, quant à trouver ce terme en langue vernaculaire ou une traduction valide en hébreu sous la plume des juifs eux-mêmes de manière assez fréquente, il faut attendre la fin du $\mathrm{XV}^{\mathrm{e}}$ siècle, dans un contexte de conversion au christianisme et non plus dans celui de l'affirmation fière, au XIII ${ }^{\mathrm{e}}$ siècle, d'une culture et d'une religion. S'il s'agissait de rechercher les origines médiévales d'un quelconque «sentiment national » ou d'une ethnogenèse juive qui s'ancrerait dans un passé antique glorieux pour rassembler un peuple autour d'une origine, d'une langue et même d'un territoire - quand bien même il serait mal «territorialisé »- le médiéviste doit se taire ${ }^{2}$. En effet, redisons ce truisme, les juifs médiévaux ne s'identifient pas à un territoire commun, ne parlent ni hébreu ni une langue vernaculaire spécifique ${ }^{3}$. L'anthroponymie, l'histoire même ne nomment pas une origine commune mais plutôt des parcours complexes de communautés juives particulières et localisées précisément en Occident. Les juifs qui habitent les terres méridionales et les royaumes ibériques ont migré depuis l'Angleterre, la Gascogne, le royaume de France, le Languedoc, al-Andalus. Leurs sentiments d'appartenance sont multiples, leur position sociale, leurs modes de vie variables, leurs solidarités fort relatives.

Mais s'il n'est pas de «nation juive», il n'est pas plus de «nation » chez les chrétiens. Il y a plutôt des nations, aux contours

1. Nation et nations au Moyen Âge, XLIV ${ }^{\text {e }}$ Congrès de la Société des Historiens Médiévistes de l'Enseignement Supérieur Public (SHMESP) (Prague, 23 mai - 26 mai 2013), Paris, 2014.

2. Claude Denjean, Juliette Sibon et Claire Soussen, «La nation juive à la fin du Moyen Âge. Mythe ou réalité ? Fantasme ou utopie ? », Nation et nations au Moyen Âge, op.cit, p. 287-298.

3. Malgré l'idée reçue si prégnante qu'on la retrouve encore dans la conclusion de Patrick Gilli, Nation et nations au Moyen Âge, op. cit., p. 333, qui cite pourtant l'article sur ce sujet ! 
flous et fluctuants ${ }^{4}$. Les difficultés que nous avions éprouvées en travaillant sur ce dossier, les débats que nous avions eus, les stratégies heuristiques et discursives que nous avions adoptées se sont révélées largement partagées par nos collègues de tous horizons. La gêne occasionnée par l'emploi de cet anachronisme, la prise de risque à la fois méthodologique et politique étaient largement partagées : une nouvelle fois, la question étudiée gagnait à l'être dans le cadre d'un comparatisme élargi, encore une fois, les réponses ne se trouvaient pas seulement dans l'aire étroite de notre spécialité 5 .

Ce colloque de 2013 a également montré combien l'effort d'envisager explicitement une notion anachronique pouvait aider les médiévistes à mieux saisir les éléments constitutifs de la période qu'ils étudient ${ }^{6}$. C'est ainsi que, forte de notre travail collectif, je vais tenter de poursuivre cette réflexion en partant d'une anthropologie historique de l'économie, au sujet d'un crédit continuellement à la lisière d'une usure stéréotypée. C'est donc autour de ces notions de (bonne) foi et de confiance (fides) que nécessite l'échange marchand, dans l'espace à la fois virtuel et spatialement bien ancré qui est celui du bien commun dans la cité médiévale que je vais reprendre le propos sur les juifs et la nation. Les travaux antérieurs et ceux de ces journées ayant force libératoire quant aux lourds débats sur la nation et les juifs ${ }^{7}$, je me garderai surtout du risque de parler de cette natio qui n'est pas, en dérivant vers les notions connexes (et tout aussi piégées) qui sont l'identité originelle ou l'ethnicité.

C'est à travers une réflexion comparatiste que je chercherai à démêler la question des juifs et de la nation, sans autre prétention que celle de saisir au plus près la situation des juifs médiévaux en Méditerranée occidentale chrétienne. Définir la longue durée de

4. Ingrid Houssaye Michienzi, «La 'nation' et les milieux d'affaires florentins aux $\mathrm{XIV}^{\mathrm{e}}$ et $\mathrm{XV}^{\mathrm{e}}$ siècles ", Nation et nations au Moyen Âge, op. cit., p. 309.

5. Comme l'ont prouvé les travaux collectifs dans le cadre de l'équipe JACOV, en particulier sur la citoyenneté, l'expertise, la notion d'étranger chez le notaire. Claude Denjean et Laurent Feller, Expertise et valeur des choses au Moyen Âge. I- Le besoin d'expertise, Madrid, Éditions de la Casa de Velázquez, 2014.

6. Pierre Monnet, "Nation et nations au Moyen Âge: introductions », Nation et nations au Moyen Âge, op. cit., p. 9-34.

7. Shlomo Sand, Comment le peuple juif fut inventé ? Paris, Flammarion, Champs essais, 2010. 
l'histoire des juifs sans anachronisme, déterminer ses scansions selon un thème aussi sensible, en déjouant les pièges, serait sans aucun doute un projet fondateur mais qui devra se construire par un travail collectif de débat. Je me bornerai donc à des remarques critiques, dans un but prospectif, pour proposer un dialogue avec les autres textes de ce recueil. Je m'appuierai d'abord sur un terrain solide et déjà bien balisé, celui des nations marchandes. Les premières hypothèses naîtront de l'analyse des sources de la pratique, en élargissant la focale: je rapprocherai les conclusions des monographies consacrées à l'histoire des Italiens avec ceux portant sur les juifs pour envisager la «natio » des hommes d'affaires. Je proposerai ensuite une ouverture vers un élargissement potentiel et problématique de mes conclusions actuelles. Traditionnellement, l'histoire intellectuelle, autonome, est convoquée au début de la recherche pour établir les concepts qui pourront ensuite être retrouvés par l'histoire économique et sociale. Les difficultés heuristiques que nous venons de signaler nous conduisent ici à tenter une progression inverse.

\section{UNE NATION (IMPARFAITE), SANS LE NOM? NATION JUIVE ET NATIONS MARCHANDES}

L'une des acceptions classique du terme et de la notion de «natio» concerne les «nations» des marchands habitant des comptoirs et garantis par le statut d' « étranger ». Des thèses récentes ont repris cette question, en particulier celle d'Elisa Soldani sur les Italiens à Barcelone ${ }^{8}$, d'Ingrid Houssaye sur Majorque ${ }^{9}$ et de Diego Deleville sur les Italiens en Dauphiné ${ }^{10}$. Leurs résultats offrent la possibilité de comparaisons qui permettent de saisir exactement les enjeux et les spécificités de l'histoire des juifs. Ils apportent en effet

8. Maria Elisa Soldani, Uomini d'affari i mercanti toscani nella Barcellona del quattrocento, Barcelone, Consell Superior d'Investigacions Científiques (CSIC), 2010.

9. Ingrid Houssaye Michienzi, «La 'nation' et les milieux d'affaires florentins aux XIV et $\mathrm{XV}^{\mathrm{e}}$ siècles", Nation et nations au Moyen Âge, op. cit., p. 299309.

10. Diego Deleville, Les Italiens en Dauphiné à la fin du Moyen Âge. Crédit, finance et pouvoir, Grenoble, Presses universitaires de Grenoble («La pierre et l'écrit »), 2012. 
des éclairages sur les notions considérées comme caractéristiques de la judéité : l'exil, la nostalgie d'une patrie perdue, et présentent la situation des hommes d'affaires constituant une communauté reconnue par les pouvoirs, étrangère, mais de même religion. L'anthroponymie de ces étrangers qui peuvent acquérir d'autres citoyennetés que celle de leur origine ressemble à celle des juifs, de même que leurs activités économiques et la caricature dont ils ont fait l'objet. Pouvons-nous estimer que la place des élites marchandes et financières juives sur le marché structurant la société urbaine du bien commun serait, pour sa part, celle d'une natio, dans le sens des nations marchandes, mais qui ne serait ni étrangère ni expatriée tout en appartenant à une autre religion? Nous avons déjà démontré que les juifs étaient des citoyens d'une sorte particulière ${ }^{11}$. Dans ce cas, les juifs seraient-ils exactement une natio ? Ne seraient-ils pas une sorte de natio dont la nécessaire incomplétude couplée à l'impossible assimilation ferait tout le prix ? Ne nous méprenons pas sur le lexique utilisé qui pourrait nous conduire à une vision d'une judéité blessée et imparfaite, jamais très loin des stéréotypes antijuifs ou de conceptions essentialistes. Nous cheminons difficilement, par approximations, vers une réalité jusque-là mal nommée parce que jamais dite pour elle-même.

\section{Être d'ici et de là-bas}

Posons d'abord les principes qui définissent ces groupes sociaux et fondent notre réflexion. Ces communautés de grands marchands vivent sur un territoire dont ils ne relèvent pas politiquement tout en pouvant y exercer le droit de propriété ; leur territorialisation est particulière puisque certains jouissent au moins en partie de l'exterritorialité, alors qu'ils opèrent au sein d'un espace international ou transnational normalisé. Notons bien que ce propos doit nécessairement se restreindre à l'étude des hommes d'affaires actifs sur les marchés locaux, régionaux et internationaux. Ne préjugeons pas de sa validité pour l'aljama ou l'universitas des juifs dans son ensemble, qui nécessite une étude

11. Claude Denjean et Juliette Sibon, «Citoyenneté et fait minoritaire dans la ville. Étude comparée des juifs de Marseille, de Catalogne et des Baléares au bas Moyen Âge », Revue d'histoire urbaine, 32, décembre 2011, p. 73-100. 
sociale encore à venir et qui n'est pas possible en tout lieu car la documentation concerne ceux qui ont quelques biens.

L'insertion dans la société englobante des hommes d'affaires que caractérisent une origine et un statut spécifiques se heurte à diverses difficultés sociales, religieuses, politiques. Bien qu'agissant individuellement et sujets à des conflits internes intenses voire virulents comme le montrent les exemples étudiés par Juliette Sibon à Marseille, Elisa Soldani à Barcelone et moimême en Catalogne, ils sont considérés, se présentent et agissent comme un groupe statutaire constitué par le respect d'un double droit: celui de leur origine géographique ou de leur religion. Cela ne les empêche pas d'obtenir de manière ponctuelle et individuelle la citoyenneté de la ville où ils exercent, voire de jouer de la reconnaissance de plusieurs citoyennetés ${ }^{12}$. Leurs mours familiales où l'endogamie reste essentielle mais non nécessairement exclusive, des préférences religieuses et culturelles qui se marquent par exemple dans l'anthroponymie, dans l'exercice de la charité ou dans le choix des moyens de paiement et de la structure des fortunes, maintenues au fil de durées longues de résidence, dessinent également les contours d'un groupe identifiable et conscient de son identité, subtil acteur de stratégies de distinction ${ }^{13}$, aptes à jouer des différences créatrices de bénéfices ${ }^{14}$. Ils peuvent occuper des positions économiques circonscrites mais jamais exclusives, spécialement le prêt à intérêt, domaine sensible où ces groupes sont juxtaposés et jamais mêlés, voire en concurrence $^{15}$. La composante sociale de ces groupes s'affiche clairement. Cependant, ces diasporas ne font jamais des choix totalement divergents et différents de ceux des membres de la société majoritaire. Leur qualification répond à la revendication d'une origine ou à une appartenance réputée qui reste vague. C'est

12. Ingrid Houssaye Michienzi, «La 'nation' et les milieux d'affaires florentins aux $\mathrm{XIV}^{\mathrm{e}}$ et $\mathrm{XV}^{\mathrm{e}}$ siècles ", Nation et nations au Moyen Âge, op. cit., p. 305-307.

13. Juliette Sibon, Les Juifs de Marseille au XIV siècle, Paris, Cerf, p. 154-156, 172-176.

14. Adela Fábregas García, La familia Spinola en el reino nazarí de Granada, Granada, Universidad de Granada, 2004.

15. Comme en témoignent les ouvrages cités Diego Deleville, Juliette Sibon, Elisa Soldani et Claude Denjean, Identités juives entre ancrage et passages en Catalogne, $\mathrm{XII}^{e}-\mathrm{XV}^{e}$ siècles, Paris-Louvain, Peeters, 2015. 
donc autour de certaines décisions stratégiques et fluctuantes que leur existence se noue : un choix géographique d'abord - migrer, rester, partir, considérer l'espace lointain d'un lieu originel de la nostalgie ; un choix identitaire ensuite, qui se dit à travers les manières d'habiter, de travailler, de construire sa famille et sa fortune, de préférer des valeurs jamais totalement en opposition mais souvent en décalage avec celles de la société majoritaire et qui dessinent un cheminement qui insère en partie dans la société englobante.

\section{L'attachement à un lieu d'origine}

Examinons donc point par point ces divers éléments à valeur taxinomique. Interrogeons-nous sur l'exercice et la conscience d'une «géographie nationale » d'abord, autour d'un mythe des origines. Dans les faits, les groupes de marchands se nomment à deux niveaux, voire plus, comme en témoignent les ouvrages de Diego Deleville et Elisa Soldani. Ils sont « italiens » ou « juifs », soit une appellation générique à même de désigner par différentiation avec un groupe de natifs, mais qui supporte également un sens politique et juridique, puisque ces hommes relèvent d'un droit et d'une représentation politique propres. Mais ils sont en même temps toscan, lombard, astesan, plus rarement sefardim, tsarfatim, les appartenances s'emboîtant et se recouvrant à l'occasion. Ils sont avant tout habitants « de telle ville $»^{16}$. Nulle harmonisation, nulle nécessité de se désigner de manière univoque et uniforme, mais au contraire une grande variabilité des cascades anthropotoponymiques qui nous informent sur ces gens à l'instar des titulatures. Si l'origine qualifie les Italiens, l'origine plus ou moins récente ${ }^{17}$ ou la résidence, parfois les deux à la fois, situent les juifs. Si le regard porté sur les étrangers peut être approximatif, englobant, variable, s'il rappelle un statut juridique spécial, l'autodésignation que pratiquent ces hommes joue sur une grammaire

16. Claude Denjean, Juliette Sibon et Sandrine Victor, « Le notaire et l'étranger. Réflexion comparative sur les statuts des juifs et des chrétiens non-citoyens dans les archives notariales provençales et catalanes (XIII ${ }^{\mathrm{e}}-\mathrm{XV}^{\mathrm{e}}$ siècles) », Apparition et installation du notariat en France, notamment méridionale (XII ${ }^{e} \mathrm{XVI} I^{e}$ siècles), Colloque international d'Albi, 26, 27, 28 septembre 2012.

17. «qui fuit de ... nunc habitator ...» 
complexe qui fait appel à la qualité distinctive face à l'autre ${ }^{18}$. Ainsi est-on italien face aux Catalans, toscan à côté d'un Lombard, florentin face aux Pisans... Les chrétiens eux-mêmes savent que certains juifs aragonais sont jugés comme des gens de peu par leurs coreligionnaires catalans ${ }^{19}$. Visiblement, l'origine lointaine compte plus pour les Italiens que pour les juifs. En effet, à l'intérieur du groupe des juifs, distingue-t-on les citoyens habitatores, cives, veïns ou vecinos et les étrangers, tant à l'intérieur de la communauté que chez le notaire chrétien. Dans tous les cas, l'origine urbaine est fondatrice et connotée affectivement. Mais le conflit politique, créateur de l'exil, domine chez les Italiens alors que la ville d'origine, souvent comparée à Jérusalem, reste le premier lieu d'attachement chez les juifs. L'observation des décisions de départ et de retour va dans le même sens : c'est le jeu de factions dans la cité d'origine et la législation sur la terre d'accueil, voire les changements diplomatiques qui règlent les relations entre les deux, qui motivent les migrations des Italiens, où politique et économie sont intimement liées, selon les analyses de Diego Deleville et d'Elisa Soldani. L'impossibilité de rester chez eux ou l'essaimage de la famille ou de l'entreprise cause le départ des juifs. Dans les deux cas, le départ reste longtemps suivi d'un espoir de retour, même utopique, mais le lieu d'origine a un statut bien différent pour les uns et les autres : un Florentin rejoindra Florence, un juif, Béziers ou Séville, parfois jusque dans le désir de sépulture ${ }^{20}$. Je ne qualifierai pas la nostalgie de Jérusalem comme un fait en rapport avec la natio mais plutôt de schème constitutif eschatologique, inscrit plus dans le temps

18. Claude Denjean, «Sources et caractéristiques de l'anthroponymie juive pyrénéenne et catalane du $\mathrm{XII}^{\mathrm{e}}$ au $\mathrm{XV}^{\mathrm{e}}$ siècle », Sources et caractéristiques de l'anthroponymie juive pyrénéenne et catalane $\mathrm{du} \mathrm{XII}^{\mathrm{e}}$ au $\mathrm{XV}^{\mathrm{e}}$ siècle », Nouvelle Revue d'onomastique, Société française d'onomastique, 37-38, 2001, p. 165-181 et Juifs et chrétiens. De Perpignan à Puigcerda, XIII -XIV siècles, Perpignan, Éditions du Trabucaire, 2004, p. 191-208; Juliette Sibon, Les Juifs de Marseille au XIV siècle, Paris, Cerf, p. 227-267.

19. ACA, PQ 1301, transcrit dans Elena Lourie. "A plot wich failed? The case of the corpse found in the Jewish call of Barcelona (1301)», dans Crusade and colonization: Muslims, Christians and Jews in Medieval Aragon, Londres, Aldershot, 1990.

20. Claude Denjean, «Vacarme à la synagogue. Pratiques religieuses juives au voisinage des chrétiens ", Mélanges en l'honneur de Gérard Nahon, Danièle et Carol Iancu (éds.), Paris, Louvain, Peeters (Collection de la Revue des études juives), 2012.», p. 237-239. 
- d'un passé glorieux et d'un futur messianique - que dans l'espace. Faut-il aller jusqu'à rechercher l'expression d'une origine catalane, majorquine, allemande... chez les juifs dont l'expression tardive s'illustrerait dans les synagogues «nationales» d'après l'expulsion des Espagnes?

\section{S'insérer, s'intégrer et perdre son appartenance nationale}

Les marqueurs culturels entretiennent un rapport visible avec la question de l'origine. Tous, les Lombards, les Toscans ou les juifs nouvellement arrivés suivent les étapes classiques de l'insertion des migrants, suivant les réseaux d'interconnaissance familiale ou communautaire. Leur habitat et leurs manières d'habiter les regroupent d'abord mais ceux qui restent pénètrent dans la ville parfois dès la première génération. Ces hommes d'affaires sont polyglottes, l'emploi de leur langue, la conservation d'une anthroponymie spécifique qui les rend repérables dans un premier temps se modifient alors qu'ils se fondent dans la population à Barcelone ou à Valence. C'est alors que ceux qui demeurent plusieurs générations finissent par adopter les habitudes locales pour exercer la charité, bâtir leur maison, élire leur sépulture ou décider de leur contrat matrimonial. C'est dans le domaine du droit familial que les différences subsistent le plus longtemps. Dans tous les cas, la proximité entre les élites urbaines de la marchandise, entretenue par l'échange, l'association, l'amitié, la position communautaire comparable devient remarquable. Tous peuvent servir dans l'administration urbaine, et obtiennent une influence politique. Certains d'entre eux finissent par se dire «catalans », ne plus revendiquer d'abord leur appartenance à une natio étrangère ${ }^{21}$. L'insertion ultime passe par l'intermariage, qui, pour les juifs, ne survient qu'avec la conversion. Quant à l'obtention de certaines charges administratives et politiques, elle ne possède pas un sens univoque. Cela a été fort bien montré dans les cas savoyards et dauphinois, fort comparables à celui des juifs de la couronne

21. Elisa Soldani pour Barcelone, à comparer avec Deleville en Dauphiné. Le mêmes attitudes ont été décrites par Henri Bresc, Arabes de langue, Juifs de religion: L'évolution du judaïsme sicilien dans l'environnement latin, XII ${ }^{e}-$ $X V^{e}$ siècles, Paris, Éditions Bouchène, 200, Claude Denjean à Puigcerda et Juliette Sibon à Marseille. 
d'Aragon: les carrières des Lombards bien en cour peuvent correspondre à un abandon de l'appartenance nationale d'origine ou au contraire en dépendre. La situation dépend de la relation entre le prince et ses sujets.

\section{DES CARACTÈRES ÉCONOMIQUES DE L'APPARTENANCE NATIONALE}

Nous venons de comprendre l'appartenance à une natio comme l'expression d'une différence statutaire (un droit différent), politique (disposer d'un gouvernement en état de négocier, être représenté, faire ou non partie du corps politique) et culturelle (montrer une convergence de valeurs) qui séparent des nations de ceux au milieu desquels elles vivent. Cependant l'essentiel, du point de vue des nations marchandes, se joue sur le marché. Le marché est l'espace social où l'harmonie doit jouer des différences, suivant un flux incessant d'échanges qui rompt un équilibre à rétablir sans cesse : si les enluminures étaient animées, on verrait sans doute trembler la balance du métier de changeur. C'est un espace abstrait et commun, partagé, accepté collectivement, garanti juridiquement par tous, quelles que soient leur loi et leur religion. On y joue de l'asymétrie des prix, des valeurs, de l'information. Les agents font en sorte de trouver des règles acceptées par tous pour se garantir contre le risque. On y joue donc de l'inégalité et de la différence, mais résolues en équilibre grâce aux valeurs d'humanité et de fides ${ }^{22}$. L'absence de spécialisation nationale généralisable a été largement démontrée. Par contre, l'assimilation simplificatrice jusqu'au stéréotype entre Lombards, Cahorsins ou juifs et l'usure est trop connue. Rappelons que ces groupes ne sont pas cantonnés dans le crédit mais qu'ils y sont surreprésentés et en sont publiquement chargés, surtout dans les pays et aux époques de prohibition absolue de l'usure, comme cela a été démontré pour la Cerdagne, la Catalogne, la Savoie et le Dauphiné.

22. Valeur reconnue à Bondavin de Draguignan dans Joseph Shatzmiller, Shylock revu et corrigé. Les juifs, les chrétiens et le prêt d'argent dans la société médiévale, Paris, 2000, comme aux prêteurs catalans et aragonais qui ne pratiquent pas l'usure dans Claude Denjean, La loi du lucre. L'usure en procès dans la Couronne d'Aragon à la fin du Moyen Âge, Madrid, Casa de Velázquez, 2011. 


\section{Le jeu fluctuant des activités «nationales»}

La natio, ou plutôt une « origine nationale », serait-elle pour quelque chose dans les qualités qui conduisent à se consacrer de manière préférentielle à une activité ? En certains lieux, comme dans le royaume nasride de Grenade, l'espace du marché joue explicitement sur les différences d'appartenance à une natio ${ }^{23}$. Une nouvelle fois, c'est la politique de l'État récepteur et la nature des relations internationales qui décident de ce caractère. Dans d'autres cas, la construction d'une éthique spécifique aboutirait à des sortes de pseudo-spécialisations que l'on appuierait sur les supposées «mœurs» du groupe, selon une pensée proche de celle qui s'exprime dans les livres de voyages ou dans les traités ${ }^{24}$. D'autre part, les représentants aux assemblées médiévales sont attachés à une sorte de "préférence nationale » (défense des intérêts de producteurs natifs d'un royaume ou d'une ville) en matière de production et de commerce, à travers des lois protectionnistes par exemple, ou bien en matière d'administration ${ }^{25}$. À l'inverse, des activités mal considérées ou prohibées sont laissées à des «étrangers ». De fait, les Lombards et autres Cahorsins, issus de groupes qui ont eu l'occasion de développer les techniques de prêt, peuvent exporter ces compétences ; leur qualité d'étrangers facilite la dévolution d'activités condamnables, puisqu'ils peuvent être expulsés à volonté après avoir répondu aux besoins de la communauté qui les accueillait après signature de chartes. Les juifs appelés dans les villes italiennes à partir du XIII ${ }^{\mathrm{e}}$ siècle jouent ce rôle. Ils assuraient les secteurs jugés peu éthiques ou simplement mal rentables du crédit dans les autres royaumes, d'autant plus facilement que d'aucuns, juifs comme chrétiens, ont brandi l'argument de leur liberté d'action à ce sujet ${ }^{26}$. Plus que

23. Voir les travaux d'Adela Fábregas García, en particulier La familia Spinola en el reino nazarí de Granada, Granada, Universidad de Granada, 2004.

24. Benoît Grévin, «Usages rhétoriques et systèmes de pensée dans l'Europe du XIII ${ }^{\mathrm{e}}$ siècle », Nation et nations au Moyen Âge, op. cit., p. 143-148.

25. Cortes de los antiguos reinos de Aragón y de Valencia y Principado de Cataluña, éd. Fidel Fita et Bienvenido Oliver, Madrid, 1896-1922. Ces prohibitions se retrouvent en Castille, en Savoie, en Dauphiné. Elles concernent essentiellement la draperie, la métallurgie et l'exercice du pouvoir administratif et judiciaire.

26. Juliette Sibon et Paul Salmona éd., Saint Louis et les Juifs. Politique et idéologie sous le règne de Louis IX, Paris, Éditions du patrimoine, Centre des Monuments nationaux, 2015, p. 163-171. 
d'une préférence nationale, il s'agirait ici d'une niche économique qui pourrait être considérée comme ethnique, qui s'avère porteuse de menaces. Les hommes d'affaires de toute religion ont intégré le crédit tout naturellement dans leur polyvalence, on pourrait même dire structurellement. La spécialisation (qui resta toujours partielle et relative) est en partie subie lorsque le crédit devient une activité prééminente. Dans ce cas, les Lombards comme les juifs ont vécu le même processus exclusif qui, forçant le trait, inventait un caractère «national», celui de l'avarice et de l'usure. Après son rôle polémique chez Dante ${ }^{27}$, son expression dans le royaume de France au XIV ${ }^{\mathrm{e}}$ siècle, en Savoie ou en Dauphiné28, il reste pour longtemps attaché aux juifs. Visiblement, la «natio» est une catégorie à géométrie variable dont jouent les marchands qui cherchent à obtenir du bénéfice et les États qui doivent dire la norme, arbitrer et policer l'espace économique. Ainsi, la place des juifs lettrés, pourvus de compétences techniques professionnelles, assez riches et membres des oligarchies locales tant communautaires qu'urbaines est remarquable dans le crédit ou les autres activités de production et de financement. Ils ne disposent pourtant pas d'une capacité à l'information spécifique. Je me demande s'il ne faut pas lire de l'ironie dans certains textes hébraïques qui mettent en rapport usure et statut juridique particulier. Nous avons souligné ailleurs comment la mise en place des lois sur l'usure créait une appartenance nationale professionnelle en assimilant usure et judéité 29 . La définition technique du taux d'usure légal, l'institution du jurement sur le Décalogue et la Torah - serment personnel ou collectif des prêteurs -, la procédure judiciaire ont contribué à cette agrégation de valeurs, certes abusive mais qui s'est révélée opératoire. Ces processus ne doivent pas être simplifiés : ils se révèlent à la fois intégrateurs et porteurs en germe d'exclusion. Ils

27. Dante Alighieri, dans son Enfer, chant XII, place les usuriers (XVII) dans le septième cercle, troisième giron, après les sodomites et avant les trompeurs. La Divine Comédie, L'Enfer/Inferno (vol. I), Jacqueline Risset éd., Paris, Flammarion, 2004 [1éd. 1985].

28. Diego Deleville, Les Italiens en Dauphiné à la fin du Moyen Âge. Crédit, finance et pouvoir, Grenoble, Presses universitaires de Grenoble ( La pierre et l'écrit»), 2012, p. 237-246.

29. Claude Denjean, «La politique de Louis IX vue d'Aragon: Jacques le conquérant, l'usure et les juifs de ses royaumes », Juliette Sibon et Paul Salmona éd., Saint Louis et les Juifs. Politique et idéologie sous le règne de Louis IX, Paris, Éditions du Patrimoine, Centre des Monuments nationaux, 2015, p. 163-171. 
contribuent à la reconnaissance publique de la fides intégratrice dans le corps civique, en particulier l'universitas sous toutes ses formes (communautaire, urbaine, professionnelle). Cette catégorisation à travers ces formes de natio s'opère de manière dialectique, non linéaire. Elle ne recouvre pas l'émergence de nationalismes, surtout pas en relation avec un territoire. Elle émerge à travers le jeu des États (jusqu'à la cité) avec les forces sociales. Elle n'aboutit pas à une forme fixe mais reste fluctuante.

\section{Une agrégation nationale faible}

Puisque c'est la mise en place et l'essor de la fiscalité qui a développé le dialogue entre le prince et des sujets constitués en communautés politiques, nous avions d'abord cherché les mentions de natio juive dans les plaintes et demandes faites aux assemblées représentatives que sont les Corts de Catalunya et les Cortes d'Aragon, ou dans les protocoles initiaux des actes compilés dans les premiers registres dits Demandarum, datant du règne de Pierre le Cérémonieux ${ }^{30}$. En effet, l'établissement d'une fiscalité royale régulière a institué et réglementé un prélèvement sur les communautés juives dans la Couronne d'Aragon, formalisé un dialogue entre leurs représentants et l'administration royale, imposé et construit un sentiment d'appartenance à la communauté fiscale. Cette hypothèse a été reprise lorsque l'exemple marseillais nous a fourni une occurrence précieuse de natio dans le contexte fiscal. Cependant, si des collectae juives peuvent présenter des caractères d'une natio, les juifs auxquels s'adressent les chartes royales sont nommés «juifs de telle ville», «de telle communauté », par exemple Judeis franchis cesarauguste ${ }^{31}$, collecta, aljama Valencie...

Malgré le mythe d'une origine commune ${ }^{32}$, il est donc impossible de qualifier les juifs dans leur ensemble de natio,

30. Mathieu Zannetin, La fiscalité des juifs en Catalogne d'après les premiers registres "Demandarum », mémoire de Master 2 soutenu en 2007 à l'université de Toulouse - Le Mirail (inédit).

31. Arxiu de la Corona d'Arago, Cancelleria, D, registre 1472, f. 6 r-v.

32. Nous prendrons alors natio dans son sens étymologique qui insiste sur la naissance, l'origine commune dans le sens cicéronien ou isidorien que Bernard Guenée, L'Occident aux XIV et XV siècles. Les États, p. 117, juge valide au Moyen Âge. 
conclusion logique car significative d'un monde médiéval conçu comme un assemblage hétérogène de communautés. Il ne faut peut-être pas exagérer le principal obstacle à l'existence explicite d'une ou de plusieurs nationes juives: l'absence d'État de référence ou d'institution alternative qui représenterait les juifs dans le jeu diplomatique, à l'instar des autres nations marchandes, du moins dans l'espace occidental. Les juifs ne sont pas exactement une nation même s'ils en présentent certains caractères, pour la simple raison qu'ils appartiennent au corps politique de la Couronne. Cependant, à l'inverse des Italiens, ils ne peuvent obtenir la citoyenneté pleine et entière car ils n'appartiennent pas à l'Ecclesia chrétienne et ne souhaitent ni partager la même religion ni pratiquer l'intermariage. Une certaine altérité, une conception d'une judéité mythique pourraient conduire à les considérer comme une seule natio, mais par ailleurs leur identité se décline : catalane, aragonaise, majorquine, valencienne, de Barcelone ou de telle autre ville, qui empêche de les considérer comme une natio étrangère. En cela, ils ne sont pas absolument différents des autres nations marchandes: les Lombards en Dauphiné et Savoie, les Florentins à Barcelone se définissaient de façon semblable.

Ainsi, si les juifs, à l'instar des Italiens, peuvent pâtir d'une conception des activités économiques incluant la catégorie «nationale», en Occident ils ne pourraient pas être nommés «natio », ils seraient la nation la moins aisément identifiable. Que la catégorie de la «nation juive», que l'on trouve explicitement à l'époque moderne voire dès l'époque des conversions en masse des juifs au christianisme, n'apparaisse pas dans les textes médiévaux est chose naturelle et ne les caractérise pas spécifiquement. Cette analyse nous instruit sans doute plus sur la nature de la société médiévale que sur la judéité elle-même. Ne retrouverions-nous pas ici un autre topos, celui d'une histoire des juifs paradigmatique?

\section{LA NATION AVANT LA NATIO}

Et pourtant, nous sommes toujours tentés de reprendre la célèbre phrase de Braudel en remplaçant «civilisation» par «nation» 
et d'affirmer qu'elle existe bel et bien, cette nation juive !33 Même lorsque nous veillons à nous garder d'une reconstruction téléologique de la nation juive, même lorsque nous nous appliquons à décloisonner nos analyses et à contextualiser les faits, tout travail de recherche nous confronte à l'évidence largement partagée d'un groupe humain que des pratiques religieuses et culturelles constituent en peuple élu doté d'un sentiment d'appartenance vigoureux dans l'adversité. L'emploi constant et fondateur, du $\mathrm{XII}^{\mathrm{e}}$ au $\mathrm{XV}^{\mathrm{e}}$ siècle, du terme générique de «judei», «les juifs», pour nommer les membres de communautés juives particulières, pour qualifier statutairement des individus devant le notaire ou l'administration mais aussi pour désigner un groupe que sa religion mais aussi des origines lointaines et des conceptions différentes opposent aux chrétiens dans la polémique, nous invite à considérer un groupe clairement identifié, bien au-delà d'un statut juridique. Son origine en quelque sorte biblique sous-entend un jeu sur les filiations multiples, tant spirituelles que proprement généalogiques. Du moins la lecture généalogique n'est-elle pas génétique. Aller de ces constatations à l'évocation d'une «nation » nous pousse à un anachronisme raisonné en considérant diverses manières d'être une nation au sens actuel du terme. Nous ne pouvons pas nous arrêter aux conclusions de notre travail comparatiste, car notre analyse resterait un point particulier dans la masse des textes sur le sujet. Il nous faut tenter d'aller plus loin.

Dresser le portrait de cette évanescente nation des juifs suppose donc une exposition polyphonique. Ce mode de réflexion et d'écriture est d'autant moins exempt de dangers qu'il semble ne pas respecter les modes d'analyse et d'expression canoniques des historiens. S'il n'est pas certain que l'aveu conduise systématiquement au pardon de la faute méthodologique, il entend manifester une lucidité soucieuse de ne pas fabriquer par la

33. Fernand Braudel, La Méditerranée et le monde méditerranéen à l'époque de Philippe II, Paris, Armand Colin, 1979 (4 édition), 2 tomes, p. 135-136: « Il y a bel et bien une civilisation juive, si particulière qu'on ne lui re- connaît pas toujours le caractère de civilisation authentique. Et pourtant, elle rayonne, transmet, résiste, accepte, refuse ; elle a tous les traits que nous avons signalés à propos des civilisations. Il est vrai qu'elle n'est pas enracinée, ou plutôt qu'elle l'est mal, qu'elle échappe à des impératifs géographiques stables, donnés une fois pour toutes. C'est sa plus forte originalité, non la seule. Son corps est dispersé, éparpillé ...». 
narration une continuité fictive de l'espace historique étudié, propice à l'émergence d'une fiction historique qui favorisa justement l'émergence des nations. Négligeant en apparence la ligne qui nous a portés jusqu'ici, nous allons mettre la nation marchande en perspective. Après avoir comparé les juifs et les Italiens, nous allons confronter l'exposé nuancé que nous venons de dresser au sujet de la nation et des juifs avec la description, produite par les juifs médiévaux, d'un monde non seulement idéalisé mais structuré autour d'autres valeurs que celles du marché.

On pourrait penser que nous répondons à une question en apparence simple: comment les juifs eux-mêmes se considèrentils? Il ne s'agit pas exactement de nous appuyer sur l'historiographie médiévale telle que la bâtissent les chroniqueurs ni même de prétendre pratiquer une histoire intellectuelle. Plutôt que d'envisager la construction d'une histoire commune, issue du texte biblique puis des chroniques mémorielles médiévales, qui fédère les communautés juives dans le malheur à travers la circulation de notices et de rites du souvenir, nous préférons mener notre quête parmi les textes des bibliothèques méridionales et ibériques qui utilisent les notions et le lexique connexes à «natio $»^{34}$. En effet, nous cherchons à saisir des éléments qui ne seraient pas explicitement employés dans la construction réfléchie d'une histoire commune, travail certes utile et qui se suffirait à soi seul, mais qui nous détournerait de notre réflexion. Nous voulons repérer les éléments encore suffisamment vagues et mouvants pour autoriser des constructions de direction libre. Des notions utiles aux juifs comme aux chrétiens qui élaborent des textes dont la nature, les matériaux et le lexique sont partagés au service d'une configuration dont l'identité est bien juive, sinon proprement identitaire. Parmi les modèles littéraires répondant à ces critères, une fois exclues les chroniques et la polémique où les communautés s'affrontent, restent le roman et le récit de voyage. Le premier est d'origine chrétienne, le second naquit en Islam.

34. B. Grévin, «Usages rhétoriques et systèmes de pensée dans l'Europe du XIII ${ }^{\mathrm{e}}$ siècle », Nation et nations au Moyen Âge, op. cit., p. 137-148. 


\section{La nation juive en courtoisie : Le roman d'Esther}

Examinons le premier. Quittant le monde réel, relisons un texte de nova courtoise méridionale. En effet, le «roman » nous introduit dans une société virtuelle mais effective, ici celle du monde féodal idéal - celui de la courtoisie - et, sinon atemporel, du moins posé comme un présent éternel situé entre l'histoire biblique et le monde contemporain de son auteur comme de ses lecteurs. Dans le corpus des romans courtois, le roman d'Esther est original car il fut écrit en caractères hébraïques par un juif pour des lecteurs juifs et fut bien diffusé. Cette littérature mi-documentaire mi-imaginaire introduit le lecteur dans un espace où les valeurs de la société commune aux juifs et aux chrétiens sont reconfigurées autour d'un pôle littéraire, en décalage mais en écho avec le réel. Dans ce monde imaginaire, certaines lignes de clivage statutaires entre juifs et chrétiens disparaissent alors qu'une distinction de genre est visiblement structurante: en courtoisie, des hommes jaloux ou au contraire fort courtois maltraitent ou respectent des dames. Cet espace virtuel est le point de départ qu'il nous faut.

La nova de Crescas du Caylar, version romancée de l'histoire d'Esther qui fut, semble-t-il, très populaire durant des siècles, si on la rapproche de la tragédie provençale d'Esther imprimée en 1774, nous est parvenue tronquée dans le manuscrit 28 de la collection d'Hermann Adler. Elle nous introduit dans un monde achronique plutôt qu'anachronique, où divers peuples anciens et contemporains vivent sur le même plan. L'auteur, qui serait Crescas fils de Joseph Caslari, sans doute juif du Caylar près de Lodève ou près de Nîmes, peut-être lié à un Israël Caslari médecin d'Avignon en 1327, écrit en caractères hébraïques un texte en provençal ${ }^{35}$. Selon Paul Meyer, Crescas écrit la langue usuelle de son temps, sans grande connaissance de la métrique. Sa graphie est souvent incorrecte.

Voici une cour du roi Assuérus fort ouverte au monde entier, où on évoque les Français et leur langue, les gens de France ou d'Espagne, d'Angleterre ou de Grèce, des juifs et des Sarrasins, et où les femmes sont soit blanches, soit noires. Le lignage est le critère essentiel pour juger d'une personne. Au royaume de

35. Paul Meyer (qui a travaillé en collaboration avec A. Neubauer), Romania, XXI, 1892, p. 194-227. 
courtoisie, les rois antiques sont seigneurs, les comtés valent les royaumes, et les peuples convergent pour offrir leurs filles. La valeur du lignage ne dépend nécessairement ni de la richesse ni de la communauté ou du pays d'origine mais reste pourtant subtilement liée à cette origine. L'ordre du monde est celui où les femmes ne portent pas les chausses ${ }^{36}$, mais c'est bien la petite Esterelle qui les sauve tous, hommes et femmes, par son courage vertueux et modeste. La morale dirait que chacun doit donc rester à sa place, être lui-même : ainsi un homme ne doit pas tromper sa compagne par l'emploi d'un langage qu'il maîtriserait mal ${ }^{37}$. Le roi Assuérus, lui, ne s'intéresse qu'à la beauté la plus remarquable. Il fait preuve deux fois de grande folie, sans aucun respect du lignage. La reine, sa première épouse, avait déjà souligné combien cet homme était indigne. Mais Dieu veut que la juive Esterelle soit impératrice du pays où elle a été nourrie lorsque le roi organise la recherche d'une épouse convenable avec sagesse. Il respecte enfin sa «dona delicada » mais brûle de savoir sur quelle terre elle est née.

Dans l'espace courtois et féodal de cette nova écrite pour des juifs sur le canevas du livre d'Esther, les frontières entre les peuples, l'importance de l'origine et de la naissance, l'appartenance à un lignage forment donc le cadre intangible de la qualité, au sein d'un empire où le souverain peut être ivre et fou, mal conseillé, mais où l'ordre naturel finit par vaincre. Cet ordre naturel donne un rôle éminent à une pauvrette orpheline juive. Au-delà de la fin de l'histoire, que nous connaissons, le désordre initial du conte rappelle la valeur spirituelle de la femme, comme Castia gilos et d'autres textes de ce genre, mais ajoute à cette inversion ou correction des valeurs habituelles la supériorité du lignage juif. Le rouleau d'Esther lui-même, texte dont le sujet est le plus proche de la vie des juifs en Diaspora, faisait l'objet de commentaires directement en phase avec l'actualité et la situation des juifs ibériques du XIV siècle. Nous savons combien, pour les gens du Moyen Âge, ces récits sont vivants, lus au présent. Ainsi un commentateur aragonais rappelle-t-il qu'un peuple, Israël,

36. Idem, « que sia senhor de son ostal tot om ».

37. Idem, «Cascun parla son lengage, sia son profieg o son damanage, que non engane sa companha se es de Fransa o d'Espanha »... «e el non deu parlar frances qu'om se creiran veraiamenz q'el fos frances naturalmenz; darian li om molher francesa... » 
a conseillé les rebelles dans la révolte nommée «Union contre Pierre III » à Valence et en Aragon ${ }^{38}$ ! Au Xv ${ }^{\mathrm{e}}$ siècle, Haman est vu comme exprimant les arguments antijuifs et Esther comme une crypto-juive. Les commentateurs s'interrogent sur les raisons qui poussent Mordechai à obliger Esther à cacher son appartenance. Dans ce contexte, les «nations » des textes antiques conservent ou retrouvent une actualité structurante. Israël est une rose parmi les nations $\mathrm{s}^{39}$. Au cours des siècles, la compréhension des textes se modifie, mais elle permet sans cesse de circonscrire un espace pour le fait d'être juif, dont la durée, en quelque sorte irréductible, demeure un élément central.

Deux faits sont remarquables, contradictoires seulement en apparence. D'une part, dans l'espace de courtoisie comme dans une partie de l'histoire d'Esther, la judéité reste soit implicite, soit masquée. D'autre part, l'appartenance au peuple «des juifs » est semblable à l'appartenance au peuple «français» par exemple. Nous reconnaissons là des conceptions exprimées dans d'autres textes $^{40}$. Ainsi, l'actualisation d'un texte traditionnel ancien permettrait aux juifs de se reconnaître une appartenance à une « nation » à l'ancienne pour garantir leur avenir, sans tenir compte de l'impossible existence d'une natio juive médiévale.

\section{Peuple et nation dans le récit de voyage de Benjamin de Tudèle}

Les auteurs juifs de récits de voyage apportent eux aussi à leurs lecteurs du baume au cœur. C'est à travers l'exotisme de la description convenue de peuples lointains et de faits qui lui sont inconnus que Benjamin de Tudèle nous livre sa conception de «peuple» et de «nation» dans sa compilation ${ }^{41}$. Le principe même de son itinéraire pose comme sujet: «les juifs », réunis

38. Barry Dov Walfish, Esther in Medieval Garb: Jewish Interpretation of the Book of Esther in the Middle Ages, Albany, State University of NewYork Press, 1993.

39. Parabole où Israël parmi les nations est comme la rose au milieu des ronces, commentaires par Rav ou Raban de Lévitique 23, 3 dans la Gemara, citée par A. Cohen, Le Talmud, p. 307.

40. B. Grévin, «Usages rhétoriques et systèmes de pensée dans l'Europe du XIII ${ }^{\mathrm{e}}$ siècle », Nation et nations au Moyen Âge, op. cit., p. 137-148.

41. Je me base sur l'édition et la traduction en anglais de Marcus Nathan Adler, The Itinerary of Benjamin of Tudela. Critical Text, Translation and Commentary, Londres, 1907. 
en congrégations et en académies d'hommes adonnés à l'étude. Le terme que l'on peut traduire par nation ou peuple [עם] est rarement employé. Des marchands des «nations» du monde entier fréquentent Montpellier. Il emploie à nouveau le terme pour nommer les Valaques dans les montagnes ${ }^{42}$, invincibles, hors-laloi, qu'aucun roi ne peut dominer, qui se donnent des noms juifs et nomment les juifs leurs frères en religion. Certains disent même qu'ils sont juifs ${ }^{43}$. Nous voyons bien que suivant le chapelet des villes, les deux sens de nation qui existent alors dans les textes apparaissent : les nations des marchands présentes dans des lieux de fréquentation internationale et des peuples marginaux ou exceptionnels qui forment un groupe aisément repérable par des mœurs particulières. Quant aux juifs, malgré l'importance de la généalogie dans chaque communauté, ce n'est pas l'appartenance ethnique qui les définit et l'on trouve des juifs noirs ${ }^{44}$.

La notion de nation reste donc, au sens strict, rarement mobilisée mais apparaît à travers un vocabulaire très fluctuant, au service d'une manière nuancée de concevoir les frontières de son monde. L'espace se dessine d'une manière beaucoup moins linéaire que dans une société parfaitement territorialisée politiquement, mais grâce à des caractères culturels, où les divers lieux ne se recouvrent pas. Le champ sémantique se rapportant aux peuples et aux nations permet de signifier des différences et des ressemblances, de la proximité avec d'autres à la différence au sein de «l'entre soi ». La natio est ici non point juridique mais ressentie. L'empathie et la convergence de valeurs dessinent des frontières inattendues par cercles concentriques. Plus qu'en inventoriant des peuples ou des races dans le sens généalogique ou génétique, c'est en étant attentif aux gradients d'une communauté de valeurs autour du «nous» juif que Benjamin rappelle une unité qui reste religieuse, que repèrent intuitivement les gardiens des tombeaux saints lorsqu'ils

42. Alors que les Assassins sont un peuple (idem, p. 15-16 et p. J), comme les Druses (p. 18/29) ; à comparer avec les Édomites parmi lesquels sont les Francs, les fils d'Ammon ou Ammonites à la frontière d'Israël, p. 17, mais aussi les Genevois. La Perse et le Khuzistan sont des terres, des pays (p. 60), ainsi que les terres asiatiques plus lointaines, jusqu'à la Chine, avec leurs peuples aux mœurs originales.

43. Idem, p. 11.

44. À Ibrig, toponyme non identifié (on trouve «Candig» dans certaines éditions), Idem, p. 65. 
conduisent le touriste juif voir les vraies tombes et non pas celles qui sont montrées au quidam.

À cette notion en quelque sorte anthropologique fait écho dans les sources de la pratique l'expression d'une hiérarchie interne spatialisée, qui varie selon les lieux et n'est en rien univoque. Si l'existence originale des juifs est incontestable, il est des juifs d'ici et des juifs d'ailleurs, étrangers, qu'ils soient de Germanie ou d'Aragon à Barcelone par exemple. D'ailleurs, les mœurs de chacun semblent bien connues de tous, si l'on en croit les procès. Une nouvelle fois, aux habitudes et rituels décrits comme étant ceux «des juifs", comme ne pas cuisiner pendant le shabbat, s'abstenir de consommer du vin non cacher ou ensevelir more judaico, s'ajoutent des pratiques identifiées comme étant d'origine régionale ou sociale.

Ainsi, dans les mondes imaginaires mais vrais que reconstituent les auteurs de novas ou de récits de voyage, une nation paradoxale apparaît dont l'incontestable unité n'exclut pas la diversité.

\section{Conclusion}

Après cette réflexion réconciliant l'anthropologie économique avec l'histoire intellectuelle, je peux reprendre les conclusions de notre travail collectif, en renforcer et en nuancer certains aspects. «Nation» au Moyen Âge est bien une catégorie partagée par les juifs et les chrétiens occidentaux qui n'est ni exactement juridique, ni religieuse, ni politique, ni identitaire mais une nébuleuse formée autour d'acceptions diverses du terme, où valeurs et politique se conjuguent dans la définition d'un «nous» revendiqué. Elle permet de classer des hommes dans des catégories opératoires que l'on arrange, et avec lesquelles on s'arrange, au gré des besoins. L'origine géographique et ses expressions culturelles comptent alors pour beaucoup. Elle trace des frontières entre des groupes, là où les frontières politiques ne sont pas déterminées. Elle fédère une communauté, la met en scène. Cette notion n'est pas exclusive d'autres notions définissant d'autres types de communautés parfois proches: terre et royaume, universitas... Elle n'a pas nécessairement vocation à avoir les mêmes bornes que le corps politique, que l'État, que l'Ecclesia ou la Synagoga. Ses 
expressions les plus communes sont marquées par les acceptions antiques ou mal définies qui tirent la natio vers le lignage, la raça et leur expression visible : les mœurs. Dans ce cas, des éléments d'une ethnogenèse sont prêts à servir un propos qui fut celui de la période moderne.

Pour les juifs, la nation exista dans un passé historique, elle est porteuse d'un sens eschatologique et en cela, elle se saisit assez naturellement pour ne pas avoir à devenir effective dans la vie sociale et politique. Cet état de fait ne correspond ni à des champs qui opposeraient la communauté à la société englobante, le privé au public, l'échange marchand au religieux. Ces systèmes d'opposition binaires n'ont pas vraiment de sens dans le monde médiéval. Cette natio évanescente, celle des juifs, se définit comme un des éléments de cet espace hétérogène et pourtant organique qui caractérise la société médiévale. Si les catégories anachroniques nous aident à penser, c'est en ce qu'elles nous obligent à ne pas plaquer nos systèmes sur ces sociétés exotiques que sont les mondes médiévaux, à toujours penser au pluriel. Dans les villes médiévales, les juifs ne sont ni des étrangers ni des minoritaires. Les juifs sont une catégorie comparable à celle des femmes, ou plutôt du féminin.

claude.denjean@sfr.fr 